\title{
WASP-38b: a transiting exoplanet in an eccentric, 6.87d period orbit ${ }^{\star}$
}

\author{
S. C. C. Barros ${ }^{1}$, F. Faedi ${ }^{1}$, A. Collier Cameron ${ }^{2}$, T. A. Lister $^{3}$, J. McCormac ${ }^{1}$, D. Pollacco ${ }^{1}$, E. K. Simpson ${ }^{1}$,

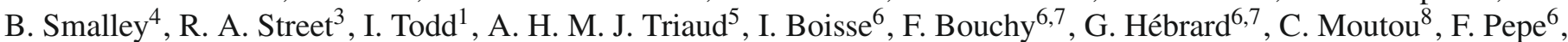 \\ D. Queloz ${ }^{6}$, A. Santerne ${ }^{8}$, D. Segransan ${ }^{6}$, S. Udry ${ }^{6}$, J. Bento ${ }^{9}$, O. W. Butters ${ }^{10}$, B. Enoch $^{2}$, C. A. Haswell ${ }^{11}$, \\ C. Hellier ${ }^{4}$, F. P. Keenan ${ }^{1}$, G. R. M. Miller ${ }^{2}$, V. Moulds ${ }^{1}$, A. J. Norton ${ }^{11}$, N. Parley ${ }^{2}$, I. Skillen ${ }^{12}$, C. A. Watson ${ }^{1}$, \\ R. G. West ${ }^{10}$, and P. J. Wheatley ${ }^{9}$
}

\footnotetext{
1 Astrophysics Research Centre, School of Mathematics \& Physics, Queen's University Belfast, University Road, Belfast, BT7 1NN, UK e-mail: s.barros@qub.ac.uk

2 SUPA, School of Physics \& Astronomy, University of St Andrews, North Haugh, St Andrews KY16 9SS, UK

3 Las Cumbres Observatory, 6740 Cortona Drive Suite 102, Goleta, CA 93117, USA

4 Astrophysics Group, Keele University, Staffordshire, ST5 5BG, UK

5 Observatoire de Geneve, Université de Geneve, 51 Ch. des Maillettes, 1290 Sauverny, Switzerland

${ }^{6}$ Institut d'Astrophysique de Paris, UMR7095 CNRS, Université Pierre \& Marie Curie, 75014 Paris, France

7 Observatoire de Haute-Provence, CNRS/OAMP, 04870 Saint-Michel l'Observatoire, France

${ }^{8}$ Laboratoire d'Astrophysique de Marseille, Université d'Aix-Marseille \& CNRS, 38 rue Frédéric Joliot-Curie, 13388 Marseille Cedex 13, France

9 Department of Physics, University of Warwick, Coventry CV4 7AL, UK

10 Department of Physics and Astronomy, University of Leicester, Leicester LE1 7RH, UK

11 Department of Physics and Astronomy, The Open University, Milton Keynes MK7 6AA, UK

12 Isaac Newton Group of Telescopes, Apartado de Correos 321, 38700 Santa Cruz de la Palma, Tenerife, Spain
}

Received 21 September 2010 / Accepted 21 October 2010

\section{ABSTRACT}

\begin{abstract}
Aims. We report the discovery of WASP-38b, a long period transiting planet in an eccentric 6.871815 day orbit. The transit epoch is $2455335.92050 \pm 0.00074(\mathrm{HJD})$ and the transit duration is $4.663 \mathrm{~h}$.

Methods. WASP-38b's discovery was enabled due to an upgrade to the SuperWASP-North cameras. We performed a spectral analysis of the host star HD 146389/BD+10 2980 that yielded $T_{\text {eff }}=6150 \pm 80 \mathrm{~K}, \log g=4.3 \pm 0.1, v \sin i=8.6 \pm 0.4 \mathrm{~km} \mathrm{~s}^{-1}, M_{*}=1.16 \pm$ $0.04 M_{\odot}$ and $R_{*}=1.33 \pm 0.03 R_{\odot}$, consistent with a dwarf of spectral type F8. Assuming a main-sequence mass-radius relation for the star, we fitted simultaneously the radial velocity variations and the transit light curves to estimate the orbital and planetary parameters. Results. The planet has a mass of $2.69 \pm 0.06 M_{\text {Jup }}$ and a radius of $1.09 \pm 0.03 R_{\text {Jup }}$ giving a density, $\rho_{\mathrm{p}}=2.1 \pm 0.1 \rho_{\mathrm{J}}$. The high precision of the eccentricity $e=0.0314 \pm 0.0044$ is due to the relative transit timing from the light curves and the RV shape. The planet equilibrium temperature is estimated at $1292 \pm 33 \mathrm{~K}$. WASP-38b is the longest period planet found by SuperWASP-North and with a bright host $\operatorname{star}(V=9.4 \mathrm{mag})$, is a good candidate for followup atmospheric studies.
\end{abstract}

Key words. planets and satellites: detection - stars: individual: WASP-38 - techniques: photometric - techniques: radial velocities

\section{Introduction}

Transiting planets are important because the geometry of these systems gives us a wealth of information. Photometry during transit allows us to derive the inclination of the orbit and the radii of both the host star and planet. Combining this information with radial velocity variations allows us to derive the absolute mass of the planet and, hence, the density. Even just an estimation of the bulk density gives us an insight into the composition of the planet (Guillot 2005; Fortney et al. 2007) and can be used to put constraints on planetary structure and formation models. These systems also offer a potential for measuring planetary emission spectra through occultation observations (e.g. Charbonneau et al. 2008) and we can gain an insight into the

* Photometry and RV data are only available in electronic form at the CDS via anonymous ftp to

cdsarc.u-strasbg.fr $(130.79 .128 .5)$ or via

http://cdsarc.u-strasbg.fr/viz-bin/qcat?]/A+A/525/A54 composition of planetary atmospheres using transit spectroscopy (Charbonneau et al. 2002; Vidal-Madjar et al. 2003; Swain et al. 2009).

For these reasons, there are several ground-based surveys searching for transiting exoplanets, such as HATNet (Bakos et al. 2004), TrES (Alonso et al. 2004), XO (McCullough et al. 2005) and WASP (Pollacco et al. 2006). Currently, there are also two space-based surveys: CoRoT (Baglin et al. 2006) and Kepler (Borucki et al. 2010). WASP is the most prolific of these surveys having discovered 38 of the 106 known transiting exoplanets. The WASP project consists of two robotic observatories: one in the Observatorio del Roque de los Muchachos, La Palma, Canary Islands, Spain and the other in the South African Astronomical Observatory of Sutherland, South Africa.

In this paper, we report the discovery of WASP-38b, an eccentric giant planet in a 6.87 day orbit. The candidate was identified in February 2010 in SuperWASP-North data. Radial velocity followup started at the end of March with FIES (2.6 m NOT). 
The planetary nature of the object was established with SOPHIE (1.93 m OHP) and CORALIE (1.2 m EULER) in May 2010. High precision photometry light curves were obtained with the Faulkes Telescope North (FTN) and Liverpool Telescope (LT).

WASP-38b is the 12th longest period of the 106 transiting exoplanets reported to date, and the fourth longest period of those discovered by ground-based observations. It was discovered after an upgrade to the SuperWASP-North cameras which we discuss in Sect. 2.1. Therefore, WASP-38b is an important object whose properties add to the known transiting planets parameter space.

\section{Observations}

\subsection{SuperWASP observations}

The SuperWASP-North observatory in La Palma consists of 8 cameras each with a Canon 200-mm f/1.8 lens coupled to an Andor e2v $2048 \times 2048$ pixel back-illuminated CCD (Pollacco et al. 2006). This configuration gives a pixel scale of $13.7^{\prime \prime} /$ pixel which corresponds to a field of view of $7.8 \times 7.8$ square degrees per camera.

In October 2008, we introduced an electronic focus control and we also started stabilisation of the temperature of the SuperWASP-North camera lenses. Prior to this upgrade, nighttime temperature variations affected the focal length of the lenses altering the $F W H M$ of stars. This introduced trends in the data (mimicking partial transits), especially at the beginning and end of the night when the temperature variation is more extreme. These effects are not corrected by our detrending algorithms SYSREM (Tamuz et al. 2005) and FTA (Kovács et al. 2005) because they are position-dependent and do not affect all stars in the same manner. To reduce this source of systematic noise, heating strips were placed around each lens so that their temperature is maintained above ambient at 21 degrees. Besides the stabilisation of the temperature we also significantly improved the focus of each of the lenses, which now can be done remotely. This upgrade was successful and proved very important for the discovery of WASP-38b.

The field containing WASP-38 (HD 146389/BD+10 2980 $\alpha=16: 15: 50.36 \delta=+10: 01: 57.3)$ was observed in the period between 2008-03-29 and 2008-06-30 by camera 144 (3777 points) and camera 145 (3278 points). During this season, our transit search algorithm (Collier Cameron et al. 2006, 2007) did not detect the transit. In the following year, after the upgrade, observation of this field continued using the same cameras between 2009-03-30 and 2009-06-30. Camera 144 recorded 5920 observations and camera 145 recorded 2922. In the 2009 data, transits were detected using both cameras. The phase folded light curve using the 2009 SuperWASP data of WASP-38 is shown in the bottom panel of Fig. 1. We also present the same for the 2008 SuperWASP data in the top panel, showing that the transit is also visible in the 2008 data. Comparing both data sets, we conclude that the somewhat higher rms $(8.7 \mathrm{mmag})$ of the 2008 data compared with the 2009 data $(6.6 \mathrm{mmag})$ prevented the detection of the transits in the first observing season.

Hence, we conclude that the upgrade was very successful in reducing the systematic noise of the SuperWASP-North cameras and allowed the discovery of a long period transiting planet. WASP-38b is the longest period transiting exoplanet found by SuperWASP-North. WASP-8b (Queloz et al. 2010) has a slightly longer period ( $P=8.16$ days) but was discovered by WASP South. Ground-based transiting surveys of exoplanets are biased towards shorter period planets due to their duty cycle and

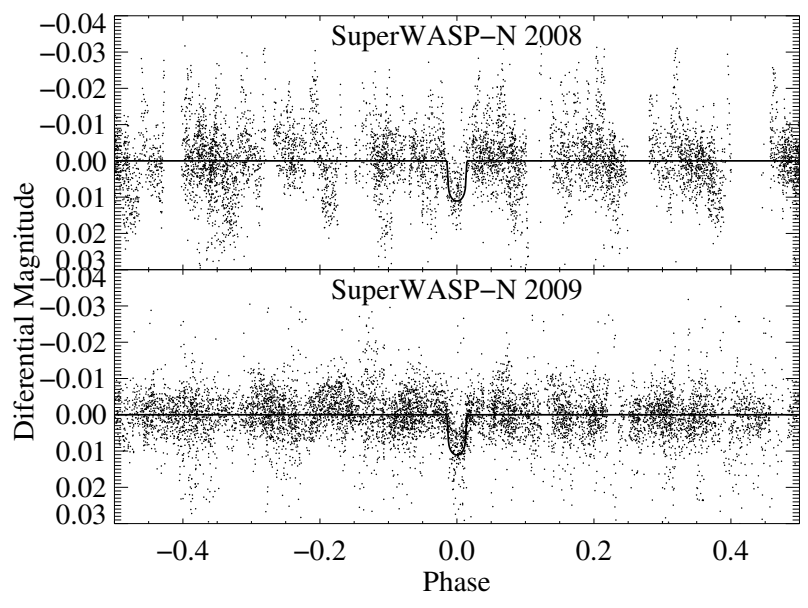

Fig. 1. SuperWASP phase folded light curve for WASP-38. On the top panel we show the 2008 data and on the bottom we show the 2009 data after the upgrade.

shorter transits. Reducing the systematic noise will be important in the discovery of long period and/or smaller radii planets.

\subsection{Spectroscopic followup}

The first radial velocity measurements of WASP-38 were taken with the Fibre-Fed Echelle Spectrograph (FIES) mounted on the $2.56 \mathrm{~m}$ Nordic Optical Telescope in La Palma. FIES was used in medium resolution mode $(R=46000)$ with simultaneous ThAr wavelength calibration. Two observations were made on the nights of 2010-03-29 and 2010-03-30. On 2010 June 08 further nine observations were taken close to phase zero but out-oftransit. The observations were reduced with the FIEStool package and cross-correlated with a high signal-to-noise spectrum of the Sun to obtain the radial velocities.

The planetary nature of WASP-38b was established with SOPHIE mounted on the $1.93 \mathrm{~m}$ telescope of the Observatoire de Haute Provence (Perruchot et al. 2008; Bouchy et al. 2009) and CORALIE on the $1.2 \mathrm{~m}$ Swiss Euler telescope in La Silla (Baranne et al. 1996; Queloz et al. 2000; Pepe et al. 2002). Ten measurements were taken by SOPHIE and 16 by CORALIE between 2010 April and July, both achieving a signal-to-noise ratio of 30 . The data was reduced with the SOPHIE and CORALIE pipelines, respectively. The radial velocity errors account for the photon noise plus known systematics in the high efficiency mode (Boisse et al. 2010).

The radial velocity measurements are given in Table 1 . In Fig. 2, we show the phase folded radial velocities from FIES (squares), SOPHIE (triangles) and CORALIE (circles). We superimpose the best fit Keplerian model described in Sect. 3.2. In the same figure we show the residuals from the Keplerian model which show no long term trend. The semi-amplitude of the radial velocities is $\sim 250 \mathrm{~m} \mathrm{~s}^{-1}$ consistent with a $2.7 M_{\text {Jup }}$ planet in a slightly eccentric orbit.

A bisector span analysis was performed on the SOPHIE and CORALIE data and is shown in Fig. 3. The bisector span shows no significant variation nor correlation with the radial velocities. This suggests that the radial velocity variations are mainly due to Doppler shifts of the stellar lines rather than stellar profile variations due to stellar activity or a blended eclipsing binary. 


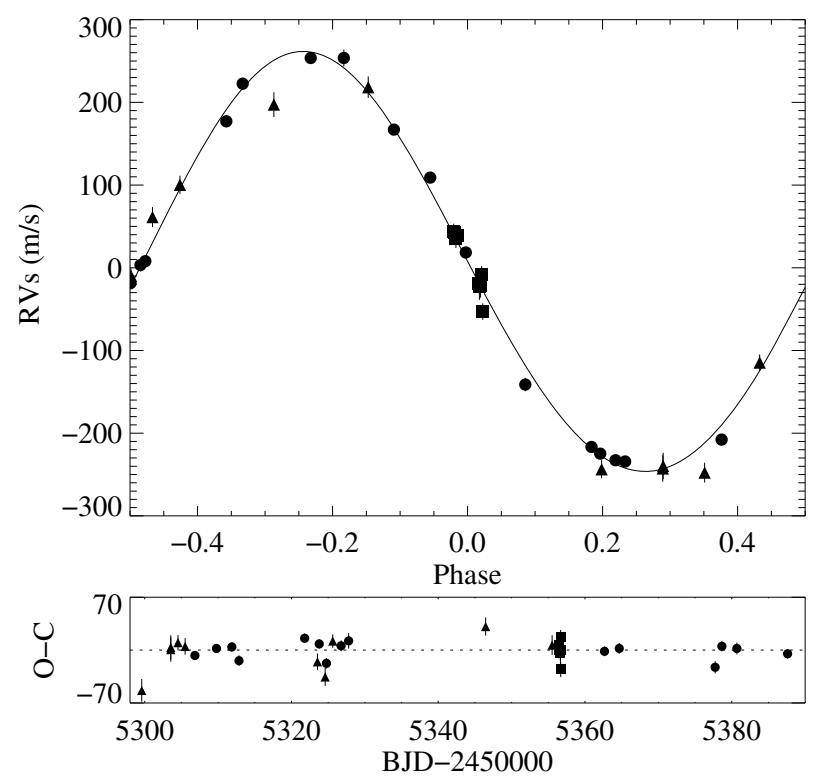

Fig. 2. Phase folded radial velocities of WASP-38 obtained with FIES (squares), SOPHIE (triangles) and CORALIE (circles). The centre-ofmass velocity for each data set was subtracted from the RVs. We also show the residuals from the orbital fit against time (bottom panel).

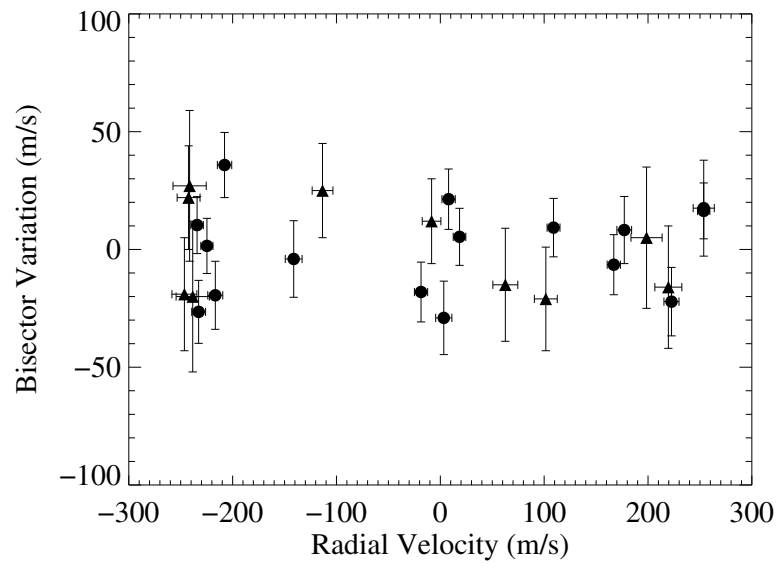

Fig. 3. Bisector span measurements for WASP-38 as a function of radial velocity for SOPHIE (triangles) and CORALIE (circles) data.

\subsection{Photometric followup}

To better constrain the system parameters, high precision transit light curves were obtained. The first photometric followup observations of WASP-38 were performed on 2010 May 19 using the LCOGT ${ }^{1} 2.0 \mathrm{~m}$ FTN located on Haleakala, Maui. The Spectral instrument was used which contains a Fairchild $4096 \times$ 4096 pixel CCD which was binned $2 \times 2$ to give $0.304^{\prime \prime}$ pixels and a field of view of $10^{\prime} \times 10^{\prime}$. Observations were taken through a Pan-STARRS $z$ filter and the telescope was defocussed during the observations to prevent saturation and to increase the exposure time and reduce the effect of scintillation. The exposure time of the observations was $20 \mathrm{~s}$. The DAOPHOT photometry package within IRAF was used to perform object detection and aperture photometry using a 16 pixel aperture radius. Differential photometry was performed relative to 23 comparison stars within the field-of-view.

\footnotetext{
1 http://lcogt.net
}

Table 1. Radial velocities of WASP-38.

\begin{tabular}{|c|c|c|c|}
\hline $\begin{array}{c}\text { BJD } \\
-2450000\end{array}$ & $\begin{array}{c}\mathrm{RV} \\
\left(\mathrm{km} \mathrm{s}^{-1}\right)\end{array}$ & $\begin{array}{c} \pm 1 \sigma \\
\left(\mathrm{km} \mathrm{s}^{-1}\right)\end{array}$ & $\begin{array}{c}V_{\text {span }} \\
\left(\mathrm{km} \mathrm{s}^{-1}\right)\end{array}$ \\
\hline \multicolumn{4}{|c|}{ FIES NOT } \\
\hline 5285.6603 & -9.678 & 0.010 & \\
\hline 5286.7164 & -9.526 & 0.008 & \\
\hline 5356.3942 & -9.800 & 0.010 & \\
\hline 5356.4056 & -9.801 & 0.009 & \\
\hline 5356.4170 & -9.808 & 0.011 & \\
\hline 5356.4284 & -9.804 & 0.010 & \\
\hline 5356.6447 & -9.862 & 0.012 & \\
\hline 5356.6561 & -9.867 & 0.015 & \\
\hline 5356.6675 & -9.865 & 0.014 & \\
\hline 5356.6788 & -9.851 & 0.010 & \\
\hline 5356.6902 & -9.896 & 0.010 & \\
\hline \multicolumn{4}{|c|}{ SOPHIE OHP } \\
\hline 5299.58942 & -9.510 & 0.015 & 0.003 \\
\hline 5303.54816 & -9.950 & 0.016 & 0.025 \\
\hline 5303.55212 & -9.947 & 0.016 & -0.022 \\
\hline 5304.53449 & -9.822 & 0.010 & 0.023 \\
\hline 5305.50502 & -9.607 & 0.011 & -0.023 \\
\hline 5323.54137 & -9.951 & 0.011 & 0.020 \\
\hline 5324.58983 & -9.955 & 0.012 & -0.021 \\
\hline 5325.62208 & -9.717 & 0.009 & 0.010 \\
\hline 5346.45645 & -9.646 & 0.012 & -0.017 \\
\hline 5355.52441 & -9.489 & 0.013 & -0.018 \\
\hline \multicolumn{4}{|c|}{ CORALIE Euler } \\
\hline 5306.836479 & -9.5406 & 0.0059 & -0.0225 \\
\hline 5309.783314 & -10.019 & 0.0059 & -0.0374 \\
\hline 5311.873800 & -9.8128 & 0.0063 & -0.0570 \\
\hline 5312.849321 & -9.6171 & 0.0071 & -0.0307 \\
\hline 5321.796623 & -9.6852 & 0.0062 & -0.0296 \\
\hline 5323.780775 & -10.0284 & 0.0061 & -0.0286 \\
\hline 5324.762705 & -10.0020 & 0.0069 & -0.0031 \\
\hline 5326.759164 & -9.5716 & 0.0073 & -0.0611 \\
\hline 5327.789065 & -9.5406 & 0.0102 & -0.0214 \\
\hline 5362.657765 & -9.6272 & 0.0064 & -0.0454 \\
\hline 5364.669083 & -10.0109 & 0.0072 & -0.0584 \\
\hline 5377.739163 & -9.9354 & 0.0081 & -0.0429 \\
\hline 5378.657251 & -10.0269 & 0.0067 & -0.0654 \\
\hline 5380.693611 & -9.7909 & 0.0078 & -0.0680 \\
\hline 5387.614500 & -9.7862 & 0.0064 & -0.0176 \\
\hline 5404.620483 & -9.7757 & 0.0061 & -0.0336 \\
\hline
\end{tabular}

Additional photometry was obtained on 2010 June 08 and 15 with a $18 \mathrm{~cm}$ Takahashi astrograph in La Palma. The CCD is an Andor $1024 \times 1024$ pixel e2v detector with 5.33" pixels and $1.5^{\circ} \times 1.5^{\circ}$ field of view. The observations were taken with the $i^{\prime}$ filter with an exposure time of $15 \mathrm{~s}$. Images were bias and dark subtracted and flat field corrected with standard IRAF packages. We performed differential photometry relative to 5 comparison stars using DAOPHOT within IRAF.

On the night of 2010 June 15 we also observed WASP-38 with the high-speed CCD camera RISE mounted on the $2.0 \mathrm{~m}$ Liverpool Telescope (Steele et al. 2008; Gibson et al. 2008). RISE has a wideband filter $\sim 500-700 \mathrm{~nm}$ which corresponds approximately to $\mathrm{V}+\mathrm{R}$. We obtained 3530 exposures in the $2 \times 2$ binning mode with an exposure time of $3.7 \mathrm{~s}$ and effectively no dead time. As usual, when using RISE for exoplanet transit observations, the telescope was defocussed by $-1.2 \mathrm{~mm}$ to spread the PSF over a larger number of pixels thereby increasing the signal-to-noise ratio. This resulted in a FWHM of $\sim 11^{\prime \prime}$. The data were reduced using the ULTRACAM pipeline (Dhillon et al. 2007) which is optimized for time-series photometry. Each frame was bias subtracted and flat field corrected. We performed 


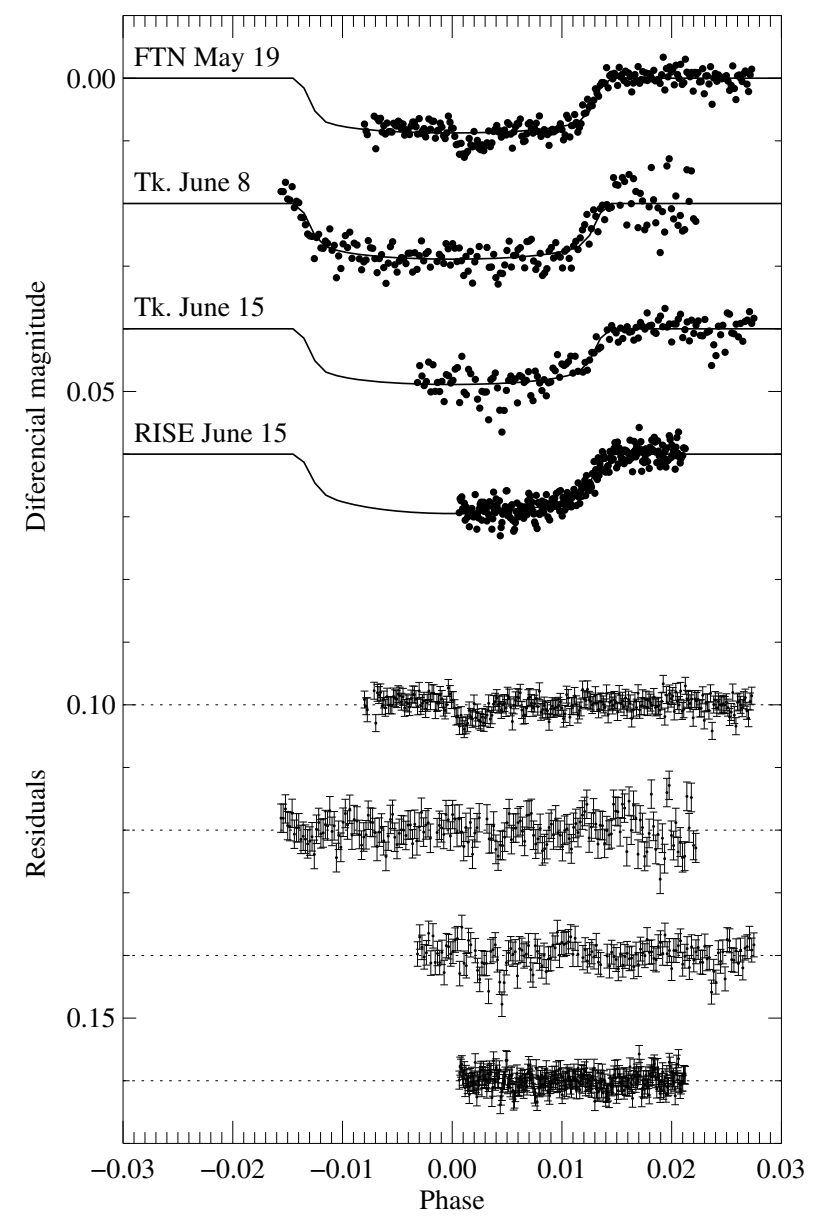

Fig. 4. Phase folded light curve for WASP-38. From top to bottom; FTN taken on the 2010 May 19, Takahashi (Tk.) astrograph taken on the 2010 June 8 and 15 and LT/RISE taken on the 2010 June 15 . We superimpose the best-fit transit model and also show the residuals for each light curve on the bottom of the figure. The data were binned and displaced vertically for clarity.

differential photometry relative to seven nearby bright stars, all checked to be non-variable. We sampled different aperture radii and chose the aperture radius that minimised the noise which turned out to be a 24 pixel aperture radius $\left(13^{\prime \prime}\right)$.

The final high precision photometric light curves are shown in Fig. 4 along with the best-fit model described in Sect. 3.2.

\section{Results and system parameters}

\subsection{Stellar parameters}

WASP-38 (HD 146389, BD+10 2980) is listed as having spectral type F8 in the HD catalogue (Cannon \& Pickering 1921). This is consistent with that implied by the value of $B-V=0.502$ given in the Tycho catalogue (Hoeg et al. 1997).

The FIES spectra were co-added to produce a single spectrum with a average signal-to-noise of around 200:1. Standard pipeline reduction products were used in the analysis.

The spectral analysis was performed using the methods given in Gillon et al. (2009). The $\mathrm{H}_{\alpha}$ line was used to determine the effective temperature ( $T_{\text {eff }}$ ), while the $\mathrm{Na}$ I D and $\mathrm{Mg}$ I $\mathrm{b}$ lines were employed as surface gravity $(\log g)$ diagnostics. Parameters obtained from the analysis are listed in Table 2. The elemental abundances were determined from equivalent width measurements of several clean and unblended lines. A value for
Table 2. Stellar parameters of WASP-38 from spectroscopic analysis.

\begin{tabular}{cc}
\hline \hline $\mathrm{RA}(\mathrm{J} 200)$ & $16: 15: 50.36$ \\
$\mathrm{DEC}(\mathrm{J} 2000)$ & $+10: 01: 57.3$ \\
$\mathrm{~V}(\mathrm{mag})$ & $9.447 \pm 0.024$ \\
$T_{\text {eff }}$ & $6150 \pm 80 \mathrm{~K}$ \\
$\log g[\mathrm{cgs}]$ & $4.3 \pm 0.1$ \\
$\xi_{\mathrm{t}}$ & $1.4 \pm 0.1 \mathrm{~km} \mathrm{~s}^{-1}$ \\
$v \sin i$ & $8.6 \pm 0.4 \mathrm{~km} \mathrm{~s}^{-1}$ \\
$\log A(\mathrm{Li})$ & $1.93 \pm 0.08$ \\
Mass $\left[M_{\odot}\right]$ & $1.16 \pm 0.09$ \\
Radius $\left[R_{\odot}\right]$ & $1.26 \pm 0.17$ \\
$\mathrm{Spectral} \mathrm{Type}$ & $\mathrm{F} 8$ \\
$\mathrm{Distance}$ & $110 \pm 20 \mathrm{pc}$ \\
{$[\mathrm{Fe} / \mathrm{H}]$} & $-0.12 \pm 0.07$ \\
{$[\mathrm{Na} / \mathrm{H}]$} & $-0.07 \pm 0.07$ \\
{$[\mathrm{Mg} / \mathrm{H}]$} & $-0.03 \pm 0.07$ \\
{$[\mathrm{Si} / \mathrm{H}]$} & $-0.01 \pm 0.04$ \\
{$[\mathrm{Ca} / \mathrm{H}]$} & $+0.00 \pm 0.13$ \\
{$[\mathrm{Sc} / \mathrm{H}]$} & $-0.03 \pm 0.16$ \\
{$[\mathrm{Ti} / \mathrm{H}]$} & $-0.06 \pm 0.12$ \\
{$[\mathrm{~V} / \mathrm{H}]$} & $-0.17 \pm 0.09$ \\
{$[\mathrm{Cr} / \mathrm{H}]$} & $-0.08 \pm 0.11$ \\
{$[\mathrm{Mn} / \mathrm{H}]$} & $-0.22 \pm 0.12$ \\
{$[\mathrm{Co} / \mathrm{H}]$} & $-0.17 \pm 0.21$ \\
{$[\mathrm{Ni} / \mathrm{H}]$} & $-0.14 \pm 0.07$ \\
\hline
\end{tabular}

Notes. Mass and radius estimate using the Torres et al. (2010) calibration. Spectral type from HD Catalogue.

microturbulence $\left(\xi_{\mathrm{t}}\right)$ was determined from Fe I using the method of Magain (1984). The quoted error estimates include that given by the uncertainties in $T_{\text {eff }}, \log g$ and $\xi_{\mathrm{t}}$, as well as the scatter due to measurement and atomic data uncertainties.

The projected stellar rotation velocity $(v \sin i)$ was determined by fitting the profiles of several unblended Fe I lines. A value for macroturbulence $\left(v_{\mathrm{mac}}\right)$ of $4.9 \pm 0.3 \mathrm{~km} \mathrm{~s}^{-1}$ was assumed, based on the tabulation by Gray (2008), and an instrumental $F W H M$ of $0.13 \pm 0.01 \AA$ was determined from the telluric lines around $6300 \AA$. A best-fit value of $v \sin i=8.6 \pm 0.4 \mathrm{~km} \mathrm{~s}^{-1}$ was obtained.

We estimated the distance by comparing the $V$ magnitude $(V=9.447)$ taken from Tycho (Hoeg et al. 1997) with the absolute magnitude of a F8-type star from Gray (1992).

\subsection{Planet parameters}

To determine the planetary and orbital parameters, we fitted all the photometry and radial velocity measurements simultaneously. Our model is an updated version of the Markov-Chain Monte Carlo (MCMC) fitting procedure described by Collier Cameron et al. (2007) and Pollacco et al. (2008). Our global fit uses the Mandel \& Agol (2002) transit model parametrised by the transit epoch $T_{0}$, orbital period $P$, impact parameter $b$, transit duration $T_{T}$ and squared ratio of planet radius to star radius $\left(R_{\mathrm{p}} / R_{*}\right)^{2}$. For each photometric data set, we include the non-linear limb darkening coefficients for the respective filter based on the tables of Claret $(2000,2004)$. The Keplerian model for the host star's reflex motion is parametrised by the centre-of-mass velocity $\gamma$, the radial velocity amplitude $K$, the orbital eccentricity $e$ and the longitude of the periastron $w$.

The main difference in the new version of our MCMC code is that the stellar mass is no longer an input parameter and is estimated from $T_{\text {eff }}, \rho_{*}$ and $[\mathrm{Fe} / \mathrm{H}]$ using the calibration of Torres et al. (2010) as described in Enoch et al. (2010). While $T_{\text {eff }}$ and $[\mathrm{Fe} / \mathrm{H}]$ are input parameters derived from spectral fitting 
Table 3. WASP-38 system parameters.

\begin{tabular}{lc}
\hline \hline Parameter & Value \\
\hline Transit epoch $T_{0}[\mathrm{HJD}]$ & $2455335.9205 \pm 0.00074$ \\
Orbital period $P$ [days] & $6.871815_{-0.000042}^{+0.000005}$ \\
Planet/star area ratio $\left(R_{\mathrm{p}} / R_{*}\right)^{2}$ & $0.00712 \pm 0.00018$ \\
Transit duration $T_{\mathrm{T}}[$ days $]$ & $0.1942_{-0.0019}^{+0.0018}$ \\
Impact parameter $b\left[R_{*}\right]$ & $0.066_{-0.046}^{+0.093}$ \\
Orbital inclination $I$ [degrees] & $89.69_{-0.25}^{+0.30}$ \\
Stellar reflex velocity $K\left[\mathrm{~m} \mathrm{~s}^{-1}\right]$ & $253.9 \pm 2.4$ \\
Orbital semimajor axis $a[\mathrm{AU}]$ & $0.07522_{-0.00075}^{+0.00074}$ \\
Orbital eccentricity $e$ & $0.0314_{-0.0046}^{+0.0046}$ \\
Longitude of periastron $\omega[$ degrees] & $-16_{-17}^{+18}$ \\
Stellar mass $M_{*}\left[M_{\odot}\right]$ & $1.203 \pm 0.036$ \\
Stellar radius $R_{*}\left[R_{\odot}\right]$ & $1.331_{-0.025}^{+0.030}$ \\
Stellar surface gravity log $g_{*}[\mathrm{cgs}]$ & $4.250_{-0.013}^{+0.012}$ \\
Stellar density $\rho_{*}\left[\rho_{\odot}\right]$ & $0.509 \pm 0.023$ \\
Planet mass $M_{\mathrm{p}}\left[M_{\mathrm{Jup}}\right]$ & $2.691 \pm 0.058$ \\
Planet radius $R_{\mathrm{p}}\left[R_{\mathrm{Jup}}\right]$ & $1.094_{-0.028}^{+0.029}$ \\
Planet density $\rho_{\mathrm{p}}\left[\rho_{\mathrm{J}}\right]$ & $2.06 \pm 0.14$ \\
\hline
\end{tabular}

(Table 2), $\rho_{*}$ is estimated at each point in the chain directly from the light curves.

Due to the poor quality of the only complete transit of WASP-38b we imposed a main-sequence mass-radius relation for the parent star, i.e. $R_{*}=M_{*}^{0.8}$ (Seager \& Mallén-Ornelas 2003; Cox 2000) in our global fit. To better constrain the system parameters, a high precision complete transit light curve is needed. Unfortunately, due to its long transit duration there are not many full transits observable for this target and the only full transit visible from La Palma this season failed due to technical issues.

The system parameters of WASP-38 and the $1 \sigma$ uncertainties derived from the MCMC analysis are given in Table 3. WASP-38b is a $2.691 M_{\text {Jup }}$ giant planet with an eccentric $(e=$ $0.031) 6.87$ day orbit. The planet radius is $1.09 R_{\mathrm{Jup}}$, and hence, it has a high density of $2.06 \rho_{\mathrm{J}}$.

\subsection{Eccentricity}

The current version of our MCMC code uses the parameters $\sqrt{e} \cos \omega$ and $\sqrt{e} \sin \omega$ as jump parameters. This scaling allows the parameter space to be explored efficiently at small eccentricities, as recommended by Ford (2006), but ensures a uniform prior on $e$ (Collier Cameron et al. 2010, in prep.).

From our global MCMC fit we derived an eccentricity of $0.0314_{-0.0041}^{+0.0046}$ which although being very small is significant at $7 \sigma$. Given the small eccentricity we also tried fitting a circular orbit for WASP-38. The $\chi^{2}$ value for the eccentric model fit is 77 while the $\chi^{2}$ value for the circular model is 143 . The eccentric model is parametrised by six parameters: $\gamma, K, e \cos \omega$, $e \sin \omega$ and two offsets to account for the shift between the zero points of the FIES, SOPHIE and CORALIE. The first two FIES points were excluded from the fit due to contamination from the moon hence we used a total of 35 RVs. Therefore, the Lucy and Sweeney test (Lucy \& Sweeney 1971) give a $99.99 \%$ probability for the eccentric orbit.

Interestingly if we fit only the radial velocities, the eccentricity is not significantly detected and the solution is compatible with a circular orbit. A more careful analysis of our data revealed that our high sensitivity to the eccentricity comes from the timing of the transit relative to the RV curve which places a tight constraint on $e \cos \omega=0.0293 \pm 0.0036$ while $e \sin \omega$ is consistent with zero. This is contrary to the common assumption that the eccentricity is almost solely constrained by the RV curve. The transit of WASP-38 occurs $\sim 1.7 \mathrm{~h}$ earlier than what was expected from the RVs if the orbit was circular. The timing shift is consistent for all of the followup light curves.

\section{Discussion}

The newly discovered planet WASP-38b is quite similar to the other currently known long-period transiting planets. It is massive $\left(2.69 M_{\mathrm{Jup}}\right)$, has an eccentric orbit $(e=0.031)$ and does not suffer from the radius anomaly. For an updated list of the properties of these objects see Kovács et al. (2010). Only eleven out of the 106 transiting exoplanets have orbital periods longer than WASP-38b. Of these, five have been discovered by the CoRoT mission, two were found by Kepler (Holman et al. 2010), two were found in radial velocity surveys (HD 17156b, Fischer et al. 2007, Barbieri et al. 2007; and HD 80606b, Naef et al. 2001, Moutou et al. 2009, Fossey et al. 2009, Garcia-Melendo \& McCullough 2009) and the remaining three are WASP-8b, HAT-P-15b (Kovács et al. 2010) and HAT-P-17b (Howard et al. 2010). Therefore, WASP-38b is the forth exoplanet with a period longer than six days discovered in a ground-based transit survey.

The low number of transiting planets with periods longer than five days is mostly due to selection effects. It is widely known that the transit probability decreases with period. Moreover, for ground-based surveys (which are responsible for the discovery of $72 \%$ of the transiting planets), the detection probability also steeply decreases with period. This is due to the longer duty cycle of the transits and longer transit duration coupled with the restricted observing time from a single site on Earth. To increase the duty cycle of the observations, telescope networks spread in geographic latitude or space-based surveys are needed. In the case of WASP-38b it was very important to reduce the systematic noise which ultimately allowed the discovery of the planet. However, the selection effects might be hiding a real decrease of the number of planets at longer periods. In fact, from radial velocity surveys there appears to be a depletion of planets between $\sim 0.1-1$ AU (Udry et al. 2003).

The low lithium abundance, $\log A(\mathrm{Li})=1.93$ points to an age of >5 Gyr for WASP-38 (Sestito \& Randich 2005). However, it has been shown (Israelian et al. 2009) that stars with planets have an under-abundance of lithium compared with stars without planets. Therefore, in this case, the lithium abundance might be overestimating the age. In fact, if we estimate the age from the rotation period ( $\sim 7.5$ days), we obtain $\sim 1$ Gyr from Barnes (2007) (using Tycho $B-V=0.5$ ). Unfortunately, our light curves are not good enough to constrain the radius of the star. As mentioned above, we had to assume the mass-radius relation for the main-sequence in our parameters fit. Hence, we cannot use the stellar radius to calculate the isochrone age. Further observations are needed in order to better constrain the age and the evolutionary status of the star.

WASP-38b is very dense but not atypically so for a massive planet. Its equilibrium temperature is $1292 \pm 33 \mathrm{~K}$ which is quite hot for a long period planet due to its "hot" F8 host star that has a luminosity $\sim 2.4 L_{\odot}$. To receive the same flux in our solar system the planet would have to be at 0.049 AU from the Sun. WASP-38b is a "pL" class planet according to the classification of Fortney et al. (2008). Therefore, we expect an efficient redistribution of heat from the day side to the night side of the planet and no temperature inversion in the atmosphere. 
A better insight on the planet composition will require a better constraint on its age. According to the Fortney et al. (2007) models, if WASP-38b is $\sim 1$ Gyr old it might have a substantial core with a mass up to 100 Earth masses. However, if the planet is much older $(4.5 \mathrm{Gyr})$ its radius is consistent with a hydrogen/helium coreless planet. A better estimation of the radius of the planet will also help constrain its composition. Given that the star is metal poor, it would be interesting to determine the existence of a core.

The Safronov number for WASP-38b is $\sim 0.3$ (Hansen $\&$ Barman 2007). With the exception of CoRoT-4b, all the transiting planets with a period longer than WASP-38b have Safronov numbers larger than 0.3 and hence they do not belong to either of the classes proposed by Hansen \& Barman (2007).

Due to its long period it is not surprising that WASP-38b is slightly eccentric. As discussed above, the eccentricity however small is significant. The circularization timescale is given by (Goldreich \& Soter 1966; Bodenheimer et al. 2001):

$\tau_{\mathrm{CIR}} \approx 0.63\left(\frac{Q_{\mathrm{p}}}{10^{6}}\right)\left(\frac{M_{\mathrm{p}}}{M_{\mathrm{Jup}}}\right)\left(\frac{M_{\odot}}{M_{*}}\right)^{3 / 2}\left(\frac{a}{10 R_{\odot}}\right)^{13 / 2}\left(\frac{R_{\mathrm{Jup}}}{R_{\mathrm{p}}}\right)^{5} \mathrm{Gyr}$.

From studies of binary stellar evolution (Meibom \& Mathieu 2005) and from our Solar System (Goldreich \& Soter 1966; Peale 1999), the tidal dissipation parameter is $Q_{\mathrm{p}}=10^{5}-10^{6}$. Therefore, for WASP-38b the circularisation timescale is between $\sim 1.9-19$ Gyr which is consistent with our constraint on the age of WASP-38 and can be compared to the main-sequence lifetime for an F8 star ( $\sim$ Gyr). Therefore, depending on the value of $Q_{\mathrm{p}}$, WASP-38b's orbit might never circularise, as it appears to be at the limit of circularisation. All longer period transiting planets are eccentric or the eccentricity has been fixed to zero. A better constraint on the age of WASP-38 might be important to constraint the tidal dissipation parameters, so further studies are encouraged.

WASP-38 is a bright star $(V=9.4 \mathrm{mag})$ and therefore is a good candidate for followup observations. The secondary transit is predicted to be at phase $0.520 \pm 0.002, T_{0}=$ $2455456.3055 \pm 0.015$ and have a duration of $274.5 \pm 5.0 \mathrm{~min}$. Next year, the only full transit visible from La Palma is on 2011 April 21. Observations of a spectroscopic transit to measure the Rossiter-McLaughlin effect were obtained in 2010 June by one of our co-authors and will be presented elsewhere (Simpson et al. 2010, in prep.).

Acknowledgements. The WASP Consortium consists of astronomers primarily from Queen's University Belfast, St Andrews, Keele, Leicester, The Open University, Isaac Newton Group La Palma and Instituto de Astrofsica de Canarias. The SuperWASP-N camera is hosted by the Issac Newton Group on La Palma. We are grateful for their support and assistance. Funding for WASP comes from consortium universities and from the UKs Science and Technology Facilities Council. Based on observations made with the Nordic Optical Telescope, operated on the island of La Palma jointly by Denmark, Finland, Iceland, Norway, and Sweden, in the Spanish Observatorio del Roque de los Muchachos of the Instituto de Astrofisica de Canarias. Based on observations made at Observatoire de Haute Provence (CNRS), France and at the ESO La Silla Observatory (Chile) with the CORALIE Echelle spectrograph mounted on the Swiss telescope. The research leading to these results has received funding from the European Community's Seventh Framework Programme (FP7/20072013) under grant agreement number RG226604 (OPTICON). F.P.K. is grateful to AWE Aldermaston for the award of a William Penny Fellowship. The RISE instrument mounted in the Liverpool Telescope was designed and built with resources made available from Queens University Belfast, Liverpool John Moores University and the University of Manchester. The Liverpool Telescope is operated on the island of La Palma by Liverpool John Moores University in the Spanish Observatorio del Roque de los Muchachos of the Instituto de Astrofisica de Canarias with financial support from the UK Science and Technology Facilities Council. We thank Tom Marsh for the use of the ULTRACAM pipeline. S.C.C.B. is grateful to Catherine Walsh for proofreading this paper.

\section{References}

Alonso, R., Brown, T. M., Torres, G., et al. 2004, ApJ, 613, L153

Baglin, A., Auvergne, M., Boisnard, L., et al. 2006, in COSPAR, Plenary Meeting, 36th COSPAR Scientific Assembly, 36, 3749

Bakos, G., Noyes, R. W., Kovács, G., et al. 2004, PASP, 116, 266

Baranne, A., Queloz, D., Mayor, M., et al. 1996, A\&AS, 119, 373

Barbieri, M., Alonso, R., Laughlin, G., et al. 2007, A\&A, 476, L13

Barnes, S. A. 2007, ApJ, 669, 1167

Bodenheimer, P., Lin, D. N. C., \& Mardling, R. A. 2001, ApJ, 548, 466

Boisse, I., Bouchy, F., Chazelas, B., et al. 2010, in New Technologies for Probing the Diversity of Brown Dwarfs and Exoplanets, EPJ Web of Conferences

Borucki, W. J., Koch, D., Basri, G., et al. 2010, Science, 327, 977

Bouchy, F., Hébrard, G., Udry, S., et al. 2009, A\&A, 505, 853

Cannon, A. J., \& Pickering, E. C. 1921, Annals of Harvard College Observatory, 96,1

Charbonneau, D., Brown, T. M., Noyes, R. W., \& Gilliland, R. L. 2002, ApJ, 568,377

Charbonneau, D., Knutson, H. A., Barman, T., et al. 2008, ApJ, 686, 1341

Claret, A. 2000, A\&A, 363, 1081

Claret, A. 2004, A\&A, 428, 1001

Collier Cameron, A., Pollacco, D., Street, R. A., et al. 2006, MNRAS, 373, 799

Collier Cameron, A., Wilson, D. M., West, R. G., et al. 2007, MNRAS, 380, 1230

Cox, A. N. 2000, Allen's astrophysical quantities (Springer)

Dhillon, V. S., Marsh, T. R., Stevenson, M. J., et al. 2007, MNRAS, 378, 825

Enoch, B., Collier Cameron, A., Parley, N. R., \& Hebb, L. 2010, A\&A, 516, A33

Fischer, D. A., Vogt, S. S., Marcy, G. W., et al. 2007, ApJ, 669, 1336

Ford, E. B. 2006, ApJ, 642, 505

Fortney, J. J., Marley, M. S., \& Barnes, J. W. 2007, ApJ, 659, 1661

Fortney, J. J., Lodders, K., Marley, M. S., \& Freedman, R. S. 2008, ApJ, 678, 1419

Fossey, S. J., Waldmann, I. P., \& Kipping, D. M. 2009, MNRAS, 396, L16

Garcia-Melendo, E., \& McCullough, P. R. 2009, ApJ, 698, 558

Gibson, N. P., Pollacco, D., Simpson, E. K., et al. 2008, A\&A, 492, 603

Gillon, M., Smalley, B., Hebb, L., et al. 2009, A\&A, 496, 259

Goldreich, P., \& Soter, S. 1966, Icarus, 5, 375

Gray, D. F. 1992, The observation and analysis of stellar photospheres, 2nd edition (Cambridge University Press)

Gray, D. F. 2008, The Observation and Analysis of Stellar Photospheres (Cambridge University Press)

Guillot, T. 2005, Ann. Rev. Earth Planet. Sci., 33, 493

Hansen, B. M. S., \& Barman, T. 2007, ApJ, 671, 861

Hoeg, E., Bässgen, G., Bastian, U., et al. 1997, A\&A, 323, L57

Holman, M. J., Fabrycky, D. C., Ragozzine, D., et al. 2010, Science [science. 1195778]

Howard, A. W., Bakos, G. Á., Hartman, J., et al. 2010, ApJ, submitted [arXiv: 1008.3898]

Israelian, G., Delgado Mena, E., Santos, N. C., et al. 2009, Nature, 462, 189

Kovács, G., Bakos, G., \& Noyes, R. W. 2005, MNRAS, 356, 557

Kovács, G., Bakos, G. Á., Hartman, J. D., et al. 2010 [arXiv: 1005. 5300]

Lucy, L. B., \& Sweeney, M. A. 1971, AJ, 76, 544

Magain, P. 1984, A\&A, 134, 189

Mandel, K., \& Agol, E. 2002, ApJ, 580, L171

McCullough, P. R., Stys, J. E., Valenti, J. A., et al. 2005, PASP, 117, 783

Meibom, S., \& Mathieu, R. D. 2005, ApJ, 620, 970

Moutou, C., Hébrard, G., Bouchy, F., et al. 2009, A\&A, 498, L5

Naef, D., Latham, D. W., Mayor, M., et al. 2001, A\&A, 375, L27

Peale, S. J. 1999, ARA\&A, 37, 533

Pepe, F., Mayor, M., Galland, F., et al. 2002, A\&A, 388, 632

Perruchot, S., Kohler, D., Bouchy, F., et al. 2008, in SPIE Conf. Ser., 7014

Pollacco, D. L., Skillen, I., Cameron, A. C., et al. 2006, PASP, 118, 1407

Pollacco, D., Skillen, I., Collier Cameron, A., et al. 2008, MNRAS, 385, 1576

Queloz, D., Mayor, M., Weber, L., et al. 2000, A\&A, 354, 99

Queloz, D., Anderson, D., Collier Cameron, A., et al. 2010, A\&A, 517, L1

Seager, S., \& Mallén-Ornelas, G. 2003, ApJ, 585, 1038

Sestito, P., \& Randich, S. 2005, A\&A, 442, 615

Steele, I. A., Bates, S. D., Gibson, N., et al. 2008, in SPIE Conf. Ser., 7014

Swain, M. R., Vasisht, G., Tinetti, G., et al. 2009, ApJ, 690, L114

Tamuz, O., Mazeh, T., \& Zucker, S. 2005, MNRAS, 356, 1466

Torres, G., Andersen, J., \& Giménez, A. 2010, A\&A Rev., 18, 67

Udry, S., Mayor, M., \& Santos, N. C. 2003, A\&A, 407, 369

Vidal-Madjar, A., Lecavelier des Etangs, A., Désert, J., et al. 2003, Nature, 422, 143 